\title{
Alpha Adrenergic Contributions to Dysrhythmia during Myocardial Ischemia and Reperfusion ịn Cats
}

\author{
Desmond J. Sheridan, Patricia A. Penkoske, Burton E. Sobel, and \\ PETER B. CORR, Cardiovascular Division, Washington University School of \\ Medicine, St. Louis, Missouri 63110
}

A в S T R A C T Alpha compared to beta adrenergic contributions to dysrhythmias induced by left anterior descending coronary occlusion and by reperfusion were assessed in chloralose-anesthetized cats $(n=96)$. Alpha receptor blockade with either phentolamine or prazosin significantly reduced the number of premature ventricular complexes during coronary reperfusion $(321 \pm 62-14 \pm 10$ premature ventricular complexes, $P<0.001$ ), abolished early ventricular fibrillation (from $25 \%$ in controls to $0 \%$ ), and prevented the increase in idioventricular rate seen with coronary reperfusion. However, $\beta$-receptor blockade was without effect. Ventricular dysrhythmias induced by coronary occlusion alone (without reperfusion) were attenuated markedly by $\alpha$-receptor blockade under conditions in which perfusion (measured with radiolabeled microspheres) within ischemic zones was not affected. Alternative sympatholytic interventions including pretreatment with 6-hydroxydopamine to deplete myocardial norepinephrine from $8.8 \pm 1.4$ to $0.83 \pm 0.2 \mathrm{ng} / \mathrm{mg}$ protein and render the heart unresponsive to tyramine (120 $\mu \mathrm{g} / \mathrm{kg}$ ) attenuated dysrhythmias induced by both coronary occlusion and reperfusion in a fashion identical to that seen with $\alpha$-receptor blockade. Although efferent sympathetic activation induced by left stellate nerve stimulation increased idioventricular rate from $66 \pm 6$ to $144 \pm 7$ beats $/ \mathrm{min}(P<0.01)$ before coronary occlusion, this response was blocked by propranolol but not by phentolamine. In contrast, during reperfusion the increase in idioventricular rate induced by left stellate nerve stimulation (to $203 \pm 14$ ) was not inhibited by propranolol but was abolished by phentolamine (79 $\pm 10)$. Intracoronary methoxamine $(0.1 \mu \mathrm{M})$ in animals depleted of myocardial catecholamines by 6-hydroxydopamine pretreatment did not affect idioventricular rate before coronary occlusion. However, early after

Dr. Sheridan was the recipient of a British-American Heart Research Fellowship. Address reprint requests to Dr. Corr.

Received for publication 29 June 1.979 and in revised form 13 August 1.979. coronary reperfusion, methoxamine increased idioventricular rate from $33 \pm 7$ to $123 \pm 21$ beats/min ( $P$ $<0.01)$. Thus, enhanced $\alpha$-adrenergic responsiveness occurs during myocardial ischemia and appears to be a primary mediator of the electrophysiological derangements and resulting malignant dysrhythmias induced by catecholamines during myocardial ischemia and reperfusion.

\section{INTRODUCTION}

Most deaths resulting from coronary artery disease are sudden and caused by ventricular fibrillation (VF). ${ }^{1}$ Although extensive coronary atherosclerosis is characteristic, complete coronary occlusions are often absent (1). Furthermore, most survivors of primary VF exhibit no definitive evidence of myocardial infarction (2). Spasm of a macroscopically normal or partially occluded coronary artery may contribute to some of these events. In experimental animals, coronary occlusion and reperfusion are associated with distinctive malignant ventricular dysrhythmias including VF, with disparate electrophysiological mechanisms. Results obtained previously suggested reentry as an underlying mechanism of dysrhythmia during coronary occlusion, in contrast to enhanced ventricular automaticity as an underlying mechanism of dysrhythmia induced by reperfusion (3). Partial or complete reperfusion of ischemic zones may be an important cause of dysrhythmias associated with coronary artery disease when flow increases because of collateral flow or regression of spasm.

Adrenergic influences on the dysrhythmias resulting from myocardial ischemia are well recognized (4). Chronic cardiac neural ablation significantly reduces the incidence of $\mathrm{VF}$, whereas acute cardiac denervation

\footnotetext{
${ }^{1}$ Abbreviations used in this paper: LAD, left anterior descending; LVEDP, left ventricular end-diastolic pressure; PVC, premature ventricular complex (es); VF, ventricular fibrillation.
} 
has little effect suggesting that residual intramyocardial catecholamines may be important factors (5). Myocardial cyclic AMP content increases significantly after experimental coronary occlusion in temporal association with the increased incidence of ventricular dysrhythmias (6).

Both the number and affinity of $\alpha$ - and $\beta$-adrenergic receptors vary under physiological and pathophysiological conditions. For example, thyroxine alters the number of detectable $\alpha$ - and $\beta$-receptors $(7,8)$. Alteration of membrane lipids induced by phospholipase reduces the number of $\beta$-adrenergic receptors in erythrocyte membranes (9). Because phospholipase activation is characteristic of ischemic myocardium (10), possible concomitant alterations in adrenergic receptors may occur also. Physiological and pharmacological studies $(11,12)$ implicate not only $\beta$-adrenergic but also potential $\alpha$-adrenergic contributions to dysrhythmia. For example, ventricular dysrhythmias induced by central stimulation of the brain with picrotoxin appear to be mediated through $\alpha$-adrenergic mechanisms (13). The present study was performed to determine whether $\alpha$ - as well as $\beta$-adrenergic effects influence electrophysiological alterations and the resulting ventricular dysrhythmias induced by ischemia and reperfusion and to evaluate their relative importance in each setting.

\section{METHODS}

Animal preparations and hemodynamic measurements. Experiments were performed with adult cats $(n=96)$ after initial anesthesia with intramuscular ketamine $(12.5 \mathrm{mg} / \mathrm{kg})$, followed 10-15 min later by intravenous $\alpha$-chloralose (75 $\mathrm{mg} / \mathrm{kg}$ ); all surgical procedures were begun 30-45 min after $\alpha$-chloralose administration. Although ketamine has been shown to produce antiarrhythmic effects (14), its short duration of action after a single dose (25-90 s) would not be expected to impair the validity of comparisons between groups. Mechanical ventilation via an endotracheal tube was provided with a Harvard ventilator (Harvard Apparatus Co., Millis, Mass.) with a mixture of oxygen and room air to maintain arterial $\mathrm{PCO}_{2}$ at $26-29 \mathrm{~mm} \mathrm{Hg}, \mathrm{PO}_{2}$ at $>100 \mathrm{~mm} \mathrm{Hg}$, and $\mathrm{pH}$ at 7.36-7.40, values obtained in normal cats (15). Muscular relaxation was maintained with decamethonium bromide $(0.25 \mathrm{mg} / \mathrm{kg}$ i.v. $)$ at $30-\mathrm{min}$ intervals and body temperature was maintained at $37.5^{\circ} \mathrm{C}$ with an esophageal thermister probe connected to a thermostatically controled lamp. A left thoracotomy was performed by excision of ribs two through four, and the pericardium was opened and sutured to the chest wall to form a cradle. Catheters were inserted into the left femoral artery and vein for recording systemic arterial pressure and administering drugs. A Gould brush model 260 recorder (Gould Inc., Instruments Div., Cleveland, Ohio) was used to monitor multiple lead surface electrocardiograms and arterial pressure. In several animals, left ventricular $\mathrm{dP} / \mathrm{dt}$ was measured continuously after differentiation of left ventricular pressure signals obtained from a catheter inserted into the left ventricle at the apex. Mean and pulsatile aortic flow were determined with the use of a calibrated $(5 \mathrm{~mm})$ flow probe and flowmeter with electronic zero (Narco Bio-Systems, Inc., Houston, Tex.). Total peripheral resistance was calculated as described (16). Stroke work index was calculated as stroke volume index $\mathrm{X}$ (mean arterial pressure - mean left atrial pressure).

The left anterior descending (LAD) coronary artery was isolated at its bifurcation from the left main trunk, proximal to all branch points. Care was taken to avoid the pericoronary nerve coursing parallel to the artery. A 3-0 cotton suture was placed under the vessel, and polyethylene tubing was threaded over the suture. Occlusion was produced by advancing the tubing to the artery and clamping it in position. Reperfusion was induced by releasing the clamp and removing the suture.

The influence of myocardial catecholamine depletion on the dysrhythmias associated with ischemia was studied by pretreating animals $(n=13) 4 \mathrm{~d}$ before surgery with 6 -hydroxydopamine $(20 \mathrm{mg} / \mathrm{kg})$ in distilled water with ascorbic acid $(\mathrm{pH}=5.2)$ as an antioxidant (17). A single dose of propranolol $(0.5 \mathrm{mg} / \mathrm{kg})$ was given at the time of 6 -hydroxydopamine treatment to avoid manifestations of the sympathomimetic response induced acutely. Adequacy of catecholamine depletion was verified in all animals by tyramine $(120 \mu \mathrm{g} / \mathrm{kg}) \mathrm{un}$ responsiveness. In addition, myocardial catecholamines were measured in fast-frozen biopsy samples (6) from selected animals $(n=8)$.

Catecholamine assay. Biopsy samples $(200 \mathrm{mg})$ from left ventricular sites were pulverized in a steel mortar in liquid nitrogen, stored at $-60^{\circ} \mathrm{C}$, homogenized in $0.4 \mathrm{~N}$ perchloric acid and $5 \mathrm{mM}$ reduced glutathione, and centrifuged at 3,000 $\mathrm{rpm}$ for $45 \mathrm{~min}$ at $0^{\circ} \mathrm{C}$. The pellet was assayed for protein, and the supernatant fraction for catecholamine content. The catecholamine assay involved methylation of tissue catecholamines with a ${ }^{3} \mathrm{H}$-labeled methyl group from $S$-adenosylL-methionine by catechol-O-methyl transferase (18) and separation of products by thin-layer chromatography before liquid scintillation spectrometry.

Regional myocardial blood flow. Regional myocardial perfusion in ischemic and nonischemic areas was measured with radiolabeled microspheres at five intervals before, during, and after coronary occlusion. Microspheres (9 $\mu \mathrm{m}$ in diameter) tagged with ${ }^{141} \mathrm{Ce},{ }^{95} \mathrm{Nb}$, or ${ }^{85} \mathrm{Sr}$ (3M Co., St. Paul, Minn.) were injected as a bolus $(0.2 \mathrm{ml})$ into the left atrium followed by a flush with saline $(5 \mathrm{ml})$. A blood sample for reference flow was obtained from the descending aorta with a Harvard withdrawal syringe pump ( $1 \mathrm{ml} / \mathrm{min}$; Harvard Apparatus Co.). Microsphere solutions were sonicated for $3 \mathrm{~min}$ just before injection to minimize aggregation, with verification by microscopy. In preliminary experiments, calculated flow was unaffected by the order in which the microspheres were administered. At the end of each experiment, the heart was excised rapidly and rinsed in saline. Three biopsies were taken from the grossly ischemic region and three biopsies from the nonischemic region of the lateral left ventricular wall supplied by the left circumflex artery. Each biopsy was subdivided into endocardial and epicardial halves. Samples were then weighed and radioactivity in each blood and tissue sample was determined with a multi-channel gamma counter (Micromedic Systems, Horsham, Pa.). Regional blood flow in each sample was calculated by computer (Digital Equipment Corp., Marlboro, Mass.; PDP-12) with the following formula after correction for crossover of energy peaks between the three isotopes. Blood flow $(\mathrm{ml} / \mathrm{min} \cdot \mathrm{gm})=($ corrected counts in organ $) \times($ reference blood flow $) /($ reference counts $) \times($ weight of heart sample). Two types of experiments were performed. In one, flow was measured before coronary occlusion, $10 \mathrm{~min}$ after occlusion, and $30 \mathrm{~s}$ after reperfusion $(n=6)$. In the other, flow was measured before coronary occlusion, $100 \mathrm{~s}$ after reperfusion, and $5 \mathrm{~min}$ after reperfusion $(n=6)$. In all experiments, occlusion was maintained for $35 \mathrm{~min}$ before reperfusion. 
Determination of idioventricular rate and neural stimulation procedures. Intense bilateral vagal nerve stimulation was employed to induce complete atrioventricular block. Although vagal stimulation per se has been shown to modestly decrease the rate of latent ventricular pacemakers $(19,20)$, this effect would be anticipated to influence results before, as well as during and after occlusion. Bipolar platinum iridium electrodes connected to a Grass stimulator with a photoncoupled isolator (Grass Instrument Co., Quincy, Mass.) were placed distally on the cervical vagal nerve trunk after decentralization to eliminate afferent nerve activation. Cervical vagal nerve stimulation in the cat, as opposed to other species, produces a pure parasympathetic response because there are no sympathetic fibers coursing with the feline vagal nerve trunk (21). Parameters of stimulation were: 2-ms impulse duration, $5 \mathrm{~V}$, and variable frequency ranging from 10 to 25 $\mathrm{Hz}$ adjusted to produce complete atrioventricular dissociation with a constant ventricular escape rate.

In studies involving stimulation of efferent sympathetic nerves a bipolar platinum iridium electrode was placed on the distal end of the inferior or middle nerve exiting the stellate ganglion after decentralization to preclude effects of afferent activation. Stimulus characteristics were: $2-\mathrm{ms}$ duration, $8 \mathrm{~V}$, and frequency ranging from 3 to $20 \mathrm{~Hz}$. In all experiments, the right stellate ganglion was removed before isolation of the left stellate ganglion.

Regional myocardial perfusion with methoxamine. The effects of an $\alpha$-adrenergic agonist, methoxamine, were evaluated by infusing the drug or vehicle at a constant rate via the LAD coronary artery $(n=8)$. The procedure involved catheterization of the femoral artery with flow directed through a pulsatile roller pump into the proximal portion of the LAD coronary artery. The catheter $(0.045$ i.d. and 0.062 o.d.) was placed in the left femoral artery and extended through a calibrated roller pump (Polystaltic, Buchler Instruments, Inc., Fort Lee, N. J.) with the open end connected to a catheter $(0.023$ i.d. and 0.038 o.d.) to be inserted into the proximal portion of the LAD coronary artery. All catheter tubing was treated with Siliclad (Clay-Adams, Div., Parsippany, N. Y.) to prevent clotting and the animals were heparinized $(1,000 \mathrm{U} / \mathrm{kg})$. Pump flow was adjusted so that the mean pressure at the junction of the catheter and the coronary artery (P23Db transducer) was equal to mean arterial pressure. This approach permits regional administration of selected compounds via a Harvard Apparatus Co. infusion pump directly into the flow of the LAD coronary artery through a " $Y$ " in the input tubing with adequate mixing with arterial blood before entry into the coronary circulation.

Pharmacological interventions. Adequacy of doses of $\alpha$ and $\beta$-adrenergic blocking agents was assessed with both pharmacological and physiological procedures. DL-propranolol $(0.75 \mathrm{mg} / \mathrm{kg})$ was administered intravenously, in a dose previously shown in the cat to effectively attenuate the heart rate increase induced by isoproterenol $(0.1 \mu \mathrm{g} / \mathrm{kg})$ or right stellate nerve stimulation (6) without inducing direct membrane effects. Alpha adrenergic blockade was induced with either phentolamine $(5 \mathrm{mg} / \mathrm{kg})$ or prazosin $(500 \mu \mathrm{g} / \mathrm{kg})$, doses required to completely attenuate the increase $(+60 \mathrm{~mm} \mathrm{Hg})$ in mean arterial pressure induced by norepinephrine $(0.1$ $\mu \mathrm{g} / \mathrm{kg}$ ) or bilateral carotid occlusion. At the termination of each experiment, the presence of adequate $\alpha$ - or $\beta$-receptor blockade was verified with these interventions.

Statistical analysis. All measured parameters are reported as mean $\pm S E$. Premature ventricular contractions, idioventricular rates, and results of stellate stimulation studies were analyzed by Student's $t$ test. Significance for death rate data was determined by chi-square analysis with $2 \times 2$ contingency with the Yates correction factor. Hemodynamic data were analyzed with a $4 \times 12$ analysis of variance, computed with a statistics analysis system (22). Mean values for each parameter for each time period were compared with $t$ tests in which the pooled estimate of the error term was used. $P$ values of $<0.05$ were considered significant for differences.

\section{RESULTS}

Because the most striking results were obtained under conditions in which myocardial reperfusion was induced, they will be presented before results obtained during sustained occlusion.

Dysrhythmias induced by reperfusion. The feline preparation used in these studies exhibits reproducible ventricular dysrhythmia after proximal LAD coronary occlusion with a consistent onset time, duration, and overall mortality caused by $\operatorname{VF}(3,6,16)$. Because the dysrhythmia induced by coronary occlusion abates completely within $35 \mathrm{~min}$, all studies involving reperfusion were performed beginning at this time. Characteristics of the dysrhythmias induced by coronary reperfusion after 35-min occlusions are shown in Fig. 1. In control animals the total number of premature ventricular complexes (PVC) elicited by reperfusion were $321 \pm 62$, and 9 of 35 animals died from VF. In contrast, phentolamine-treated animals $(5 \mathrm{mg} / \mathrm{kg}$ ) exhibited a dramatic reduction in the total number of PVC $(14 \pm 10)$ and decreased death caused by VF ( 0 of 29$)$. To ascertain whether the effects of phentolamine were nonspecific, an additional group of animals was treated with prazosin $(500 \mu \mathrm{g} / \mathrm{kg})$ resulting in a similar reduction of PVC (44.3 \pm 20.3$)$ and mortality (0 of 9) (Fig. 1).

An alternative sympatholytic intervention, pretreatment of animals with 6-hydroxydopamine $4 \mathrm{~d}$ before each study resulted in a reduction of ventricular norepinephrine content from $8.8 \pm 1.4$ in controls $(n=8)$ to $0.83 \pm 0.02 \mathrm{ng} / \mathrm{mg}$ protein in treated animals $(n=8)$. It also suppressed PVC induced by reperfusion and prevented VF; findings similar to those seen with $\alpha$ adrenergic blockade.

In contrast to the protective influence of $\alpha$-receptor blockade with either phentolamine or prazosin and of myocardial catecholamine depletion, $\beta$-receptor blockade with propranolol failed to significantly reduce either the number of PVC induced by reperfusion or the mortality and underlying VF (Fig. 1) $(n=10)$.

Idioventricular rate. The intrinsic idioventricular rate was assessed with intense bilateral efferent vagal nerve stimulation in control $(n=10)$, phentolamine$(n=8)$, and propranolol-pretreated $(n=6)$ cats (Fig. 2$)$. We have previously demonstrated that coronary reperfusion induces a rapid increase in idioventricular rate in contrast to coronary occlusion alone (3). The rapid increase in idioventricular rate seen early after reperfusion in control animals to nearly $400 \%$ of control levels was abolished in animals treated with the $\alpha$ adrenergic blocking agent, whereas $\beta$-adrenergic block- 


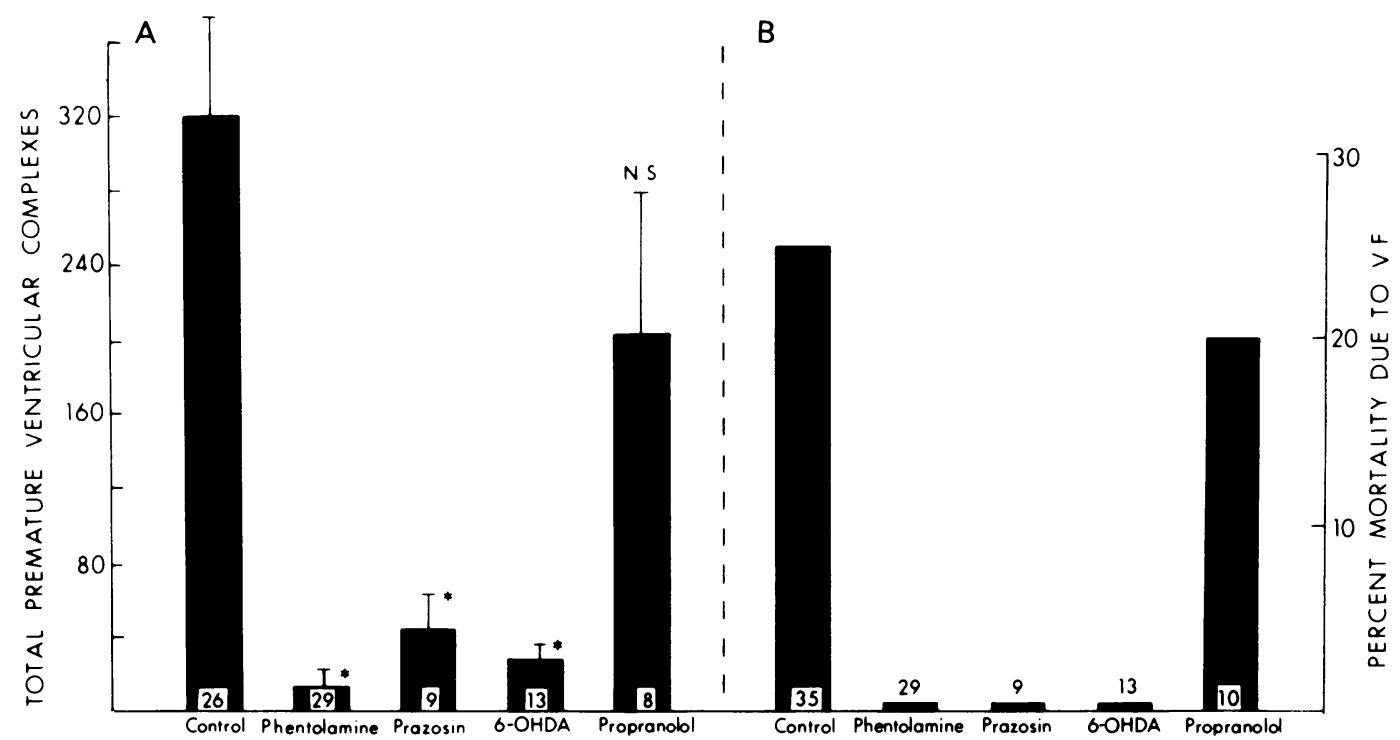

FIGURE 1 Number of PVC (A) and the percent mortality caused by VF (B) after coronary reperfusion in control animals and animals treated with either phentolamine, prazosin, 6-hydroxydopamine (6-OHDA), or propranolol. Vertical bars represent SEM, and numbers within the histograms indicate the number of animals in each group. ${ }^{*}$ indicates significant differences from control $(P<0 .() 1)$.

ade with propranolol had no discernible effect (Fig. 2). 15 min after coronary reperfusion, at a time when the dysrhythmia had abated completely, idioventricular rate had returned to control values in all three groups. Thus, $\alpha$-receptor blockade but not $\beta$-receptor blockade completely attenuated the increase in idioventricular rate associated with early reperfusion.

Resional myocardial blood flow during coronary occlusion and reperfusion. Because the protective in-

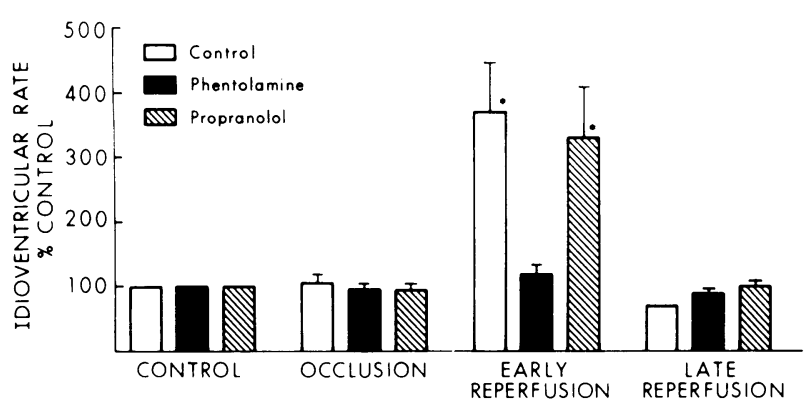

Figure 2 The idioventricular rate (percentage of control), determined by efferent vagal nerve stimulation at four time periods (control; during occlusion; early reperfusion, $2 \mathrm{~min}$; and later reperfusion, $15 \mathrm{~min})$ in control animals $(n=10)$ and animals treated with either phentolamine $(n=8)$ or propranolol $(n=6)$. There were no significant differences in idioventricular rates before coronary occlusion between the three groups. The vertical bars represent the SEM. * indicates a significant increase in the idioventricular rate $(P<0.01)$. fluence of $\alpha$-receptor blockade could be secondary to enhancement of myocardial blood flow, regional perfusion was measured in both normal and ischemic regions (Fig. 3). Flow was determined separately in the outer half of each biopsy (designated epicardial) and the inner half of each biopsy (designated endocardial). Pretreatment of animals with phentolamine had no significant effect on regional flow during any of the intervals studied (Fig. 3). Furthermore, during reperfusion the magnitude and time-course of hyperemia in control and phentolamine-treated animals was similar. Although a marked disparity between ischemic and normal zone blood flow was evident with a maximum difference $100 \mathrm{~s}$ after reperfusion, alterations were similar in control and phentolamine-treated animals (Fig. 3). Thus phentolamine pretreatment did not significantly alter regional coronary flow during coronary occlusion or subsequent reperfusion.

Left stellate stimulation studies. This series of experiments was performed to determine whether reperfusion altered the adrenergic responsiveness to efferent left stellate nerve stimulation reflected by idioventricular rate. The control idioventricular rate assessed by bilateral efferent vagal nerve stimulation was $66 \pm 6$ beats/min (Fig. 4 ). The increase in idioventricular rate observed with efferent left stellate nerve stimulation before coronary occlusion was abolished by propranolol $(n=4)$ and unaffected by phentolamine $(n=4)$ indicating a $\beta$-adrenergic-mediated response 

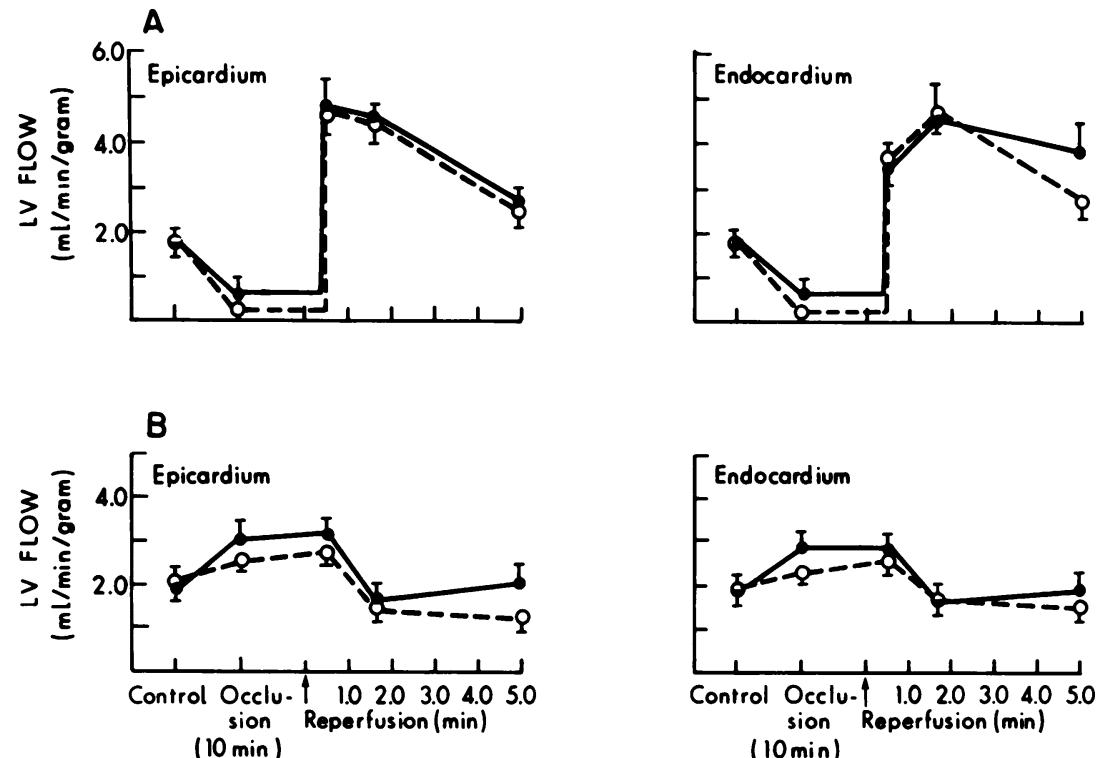

FIGURE 3 Regional left ventricular (LV) blood flow determinations in ischemic zone (A) and normal zone (B) before coronary occlusion (control), $10 \mathrm{~min}$ after coronary occlusion and $30 \mathrm{~s}$, $100 \mathrm{~s}$, and $5 \mathrm{~min}$ after coronary reperfusion in control animals $(\bigcirc)$ and animals treated with phentolamine $(O)$. Blood flow was determined in endocardial and epicardial portions of full thickness biopsies after dividing each into inner (left) and outer halves (right). Each point represents the mean of three biopsies from each zone in a minimum of six animals; vertical bars represent the SEM.

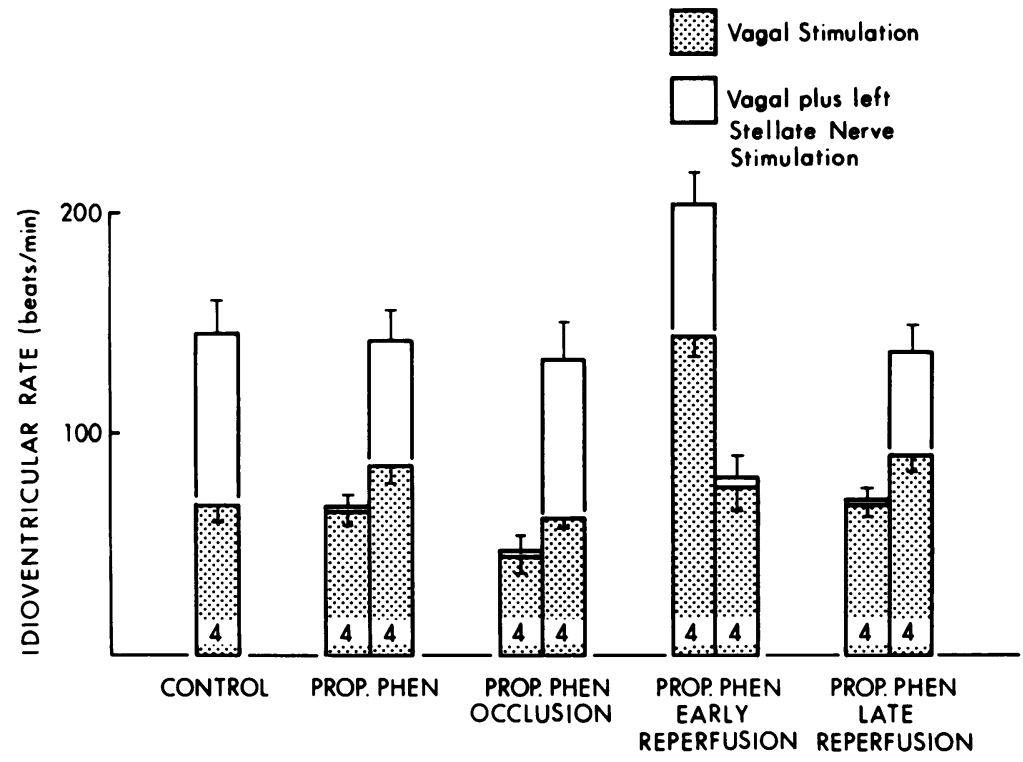

FIGURE 4 Influence of decentralized left stellate nerve stimulation on the intrinsic idioventricular rate determined by efferent vagal nerve stimulation (dotted portion of histogram). The open portion of each histogram indicates the response obtained in the presence of concomitant left stellate nerve stimulation during control periods (left) and occlusion, early reperfusion ( $2 \mathrm{~min})$ and late reperfusion ( $15 \mathrm{~min}$ ) after the ventricular dysrhythmia induced by reperfusion had abated. During each time period the response obtained in the presence of either propranolol (PROP) or phentolamine (PHEN) is shown. Vertical bars represent the SEM and the numbers within the histograms indicate the number of animals in each group. 
(Fig. 4). Phentolamine-treated animals had a slightly higher idioventricular rate before occlusion and during vagal stimulation, possibly reflecting presynaptic inhibition with overflow stimulation of $\beta$-adrenergic receptors. After coronary occlusion, propranolol again abolished the increase in idioventricular rate induced by left stellate nerve stimulation, whereas phentolamine was without effect (Fig. 4). In contrast to the $\beta$ mediated effects in the presence of control occlusion conditions, effects during reperfusion differed markedly. In propranolol-treated animals, idioventricular rate increased not only in response to reperfusion per se $(143 \pm 6)$ but also in response to combined vagal plus left stellate nerve stimulation with an additional significant increase to $203 \pm 14$. On the other hand, animals treated with phentolamine exhibited no increase in idioventricular rate during reperfusion per se $(75$ \pm 10 ) or during left stellate nerve stimulation $(79 \pm 9)$. Later during coronary reperfusion when the dysrhythmia had abated (after $15 \mathrm{~min}$ ), idioventricular rate returned to control values and $\beta$-mediated responsiveness returned (Fig. 4). Representative results demonstrating the ineffectiveness of propranolol in attenuating the increase in idioventricular rate induced by left stellate nerve stimulation $30 \mathrm{~s}$ after coronary reperfusion are illustrated in Fig. 5A. The effectiveness of phentolamine in abolishing the response to left stellate nerve stimulation $30 \mathrm{~s}$ after reperfusion is also shown in Fig. $5 \mathrm{~B}$. The alterations in $\alpha$ - and $\beta$-adrenergic responsiveness during early coronary reperfusion occurred simultaneously with the onset and duration of the dysrhythmia induced by reperfusion. Thus, coronary reper-

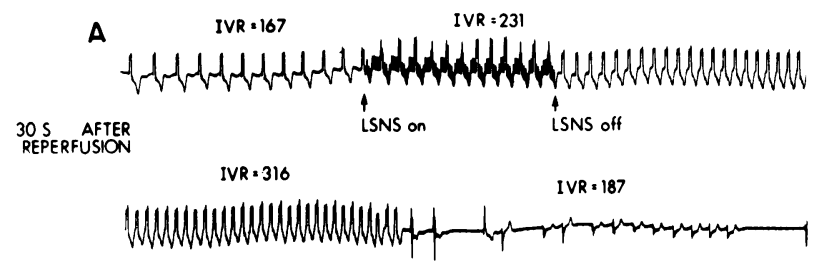

B

$$
\text { rrmprrmprrmpror }
$$

$30 \mathrm{~S}$ AFTER
REPERFUSION

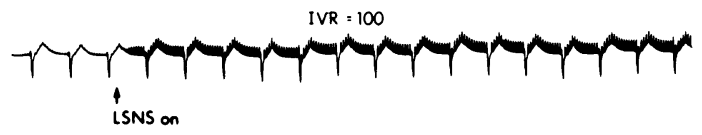

FIgure 5 Two representative experiments illustrating the alterations in the idioventricular rate (IVR) $30 \mathrm{~s}$ after coronary reperfusion, as assessed by intense bilateral vagal nerve stimulation in the presence of $0.75 \mathrm{mg} / \mathrm{kg}$ propranolol (A) or $5 \mathrm{mg} / \mathrm{kg}$ phentolamine (B). The arrows indicate the onset (on) or termination (off) of left stellate nerve stimulation (LSNS). fusion is associated with a transient alteration in $\alpha$ - and $\beta$-adrenergic responsiveness and is temporally related to the ventricular dysrhythmias induced by reperfusion.

Effects of infusions of methoxamine. To further examine adrenergic responsiveness during coronary reperfusion, methoxamine $(0.1 \mu \mathrm{M})$, an $\alpha$-adrenergic agonist, was infused directly into the left anterior descending coronary artery in animals depleted of myocardial catecholamines by prior treatment with 6 -hydroxydopamine (Fig. 6). Before abrupt interruption of coronary flow, idioventricular rate $(47 \pm 4$ beats $/ \mathrm{min})$ was not significantly altered by methoxamine $(50 \pm 2$ beats/min). In contrast, early after coronary reperfusion methoxamine significantly increased idioventricular rate $(125 \pm 22$ beats $/ \mathrm{min}$ ) compared to results in animals depleted of myocardial catecholamines and subjected to reperfusion but not given methoxamine. The increase in idioventricular rate induced by methoxamine after coronary reperfusion was maximum within $2 \mathrm{~min}$ (Fig. 6). After $10 \mathrm{~min}$, the enhanced sensitivity to methoxamine disappeared.

Dysrhythmias induced by coronary occlusion. The dysrhythmias induced during $35 \mathrm{~min}$ of coronary occlusion alone were attenuated also by $\alpha$-receptor blockade (Table I). However, in contrast to the case after reperfusion, $\beta$-receptor blockade induced an antiarrhythmic response. During coronary occlusion, $\alpha$-receptor blockade with either phentolamine or prazosin abolished mortality caused by VF compared to control animals in whom $28 \%$ died with early VF. In addition, there was a significant reduction in the number of PVC (Table I). Also, myocardial catecholamine depletion dimin-

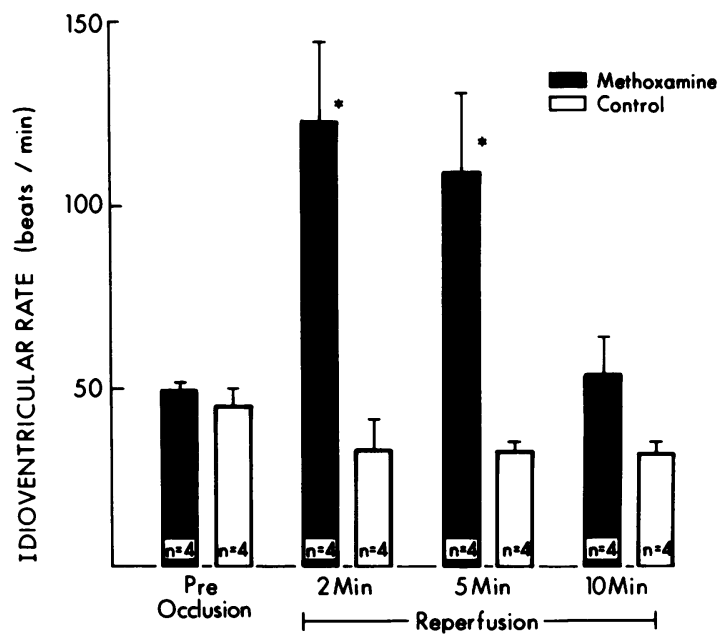

Figure 6 Influence of regional (LAD) infusion of methoxamine $(0.1 \mu \mathrm{M})$ on the idioventricular rate before coronary occlusion and at 2,5 , and $10 \mathrm{~min}$ after reperfusion in animals pretreated with 6-hydroxydopamine. Control histograms represent animals infused with the vehicle only without methoxamine. Vertical bars represent the SEM and * denotes a significant increase in the idioventricular rate $(P<0.01)$. 
TABLE I

Ventricular Dysrhythmias Induced by Coronary Occlusion

\begin{tabular}{lcr}
\hline & $\begin{array}{c}\text { Mortality caused by } \\
\text { ventricular } \\
\text { fibrillation }\end{array}$ & $\begin{array}{c}\text { Total number } \\
\text { of premature } \\
\text { ventricular } \\
\text { complexes }\end{array}$ \\
\hline Control & 14 of $50(28 \%)$ & $908 \pm 120$ \\
Phentolamine & 0 of $29(0 \%)$ & $234 \pm 73^{*}$ \\
Prazosin & 0 of $9(0 \%)^{*}$ & $280 \pm 71^{*}$ \\
6-Hydroxydopamine & 0 of $13(0 \%)^{*}$ & $83 \pm 31^{*}$ \\
Propranolol & 2 of $12(17 \%)$ & $170 \pm 60^{*}$ \\
\hline
\end{tabular}

${ }^{*} P<0.05$ in comparison to controls.

ished PVC during coronary occlusion. None of the 6hydroxydopamine-pretreated animals exhibited VF. In contrast, $\beta$-adrenergic blockade with propranolol reduced the total number of PVC but failed to reduce the incidence of $\mathrm{VF}$, a finding similar to that reported previously demonstrating mortality with $\mathrm{VF}$ after coronary occlusion in three of eight cats despite the presence of $\beta$-adrenergic blockade (6). Thus, $\alpha$-adrenergic blockade or myocardial catecholamine depletion reduces both PVC and VF associated with coronary occlusion alone. However, $\beta$-adrenergic blockade fails to significantly reduce mortality caused by VF even though it does reduce the total number of PVC (Table I).

Hemodynamics. In phentolamine- or prazosintreated animals sinus rate did not differ significantly from values in control animals (Fig. 7). Thus, the antiarrhythmic effectiveness of these agents cannot be attributed exclusively to effects on sinus rate. Animals treated with propranolol had significantly lower heart rates than controls or those treated with either phentolamine or prazosin $(P<0.05$ for each comparison).

Mean arterial pressure was significantly lower in both phentolamine- and prazosin-treated animals compared to values in controls $(P<0.05)$. Despite the marked differences in antiarrhythmic effectiveness, animals treated with propranolol demonstrated a reduction in mean arterial pressure comparable to that seen in phentolamine- and prazosin-treated cats (Fig. 7). Thus, the protective influence of $\alpha$-adrenergic blockade does not appear to depend exclusively on alterations in mean arterial pressure. Analysis of alterations in left ventricular end-diastolic pressure in the four groups revealed no significant differences during coronary occlusion (Fig. 7). However, $2 \mathrm{~min}$ after reperfusion, both phentolamine- and prazosin-treated animals demonstrated significantly lower left ventricular enddiastolic pressure (LVEDP) compared to untreated animals. Nevertheless, LVEDP remained within normal limits throughout the experiments in all animals (Fig. 7).

Neither cardiac output nor peak left ventricular $\mathrm{dP} / \mathrm{dt}$ differed significantly among any of the four groups
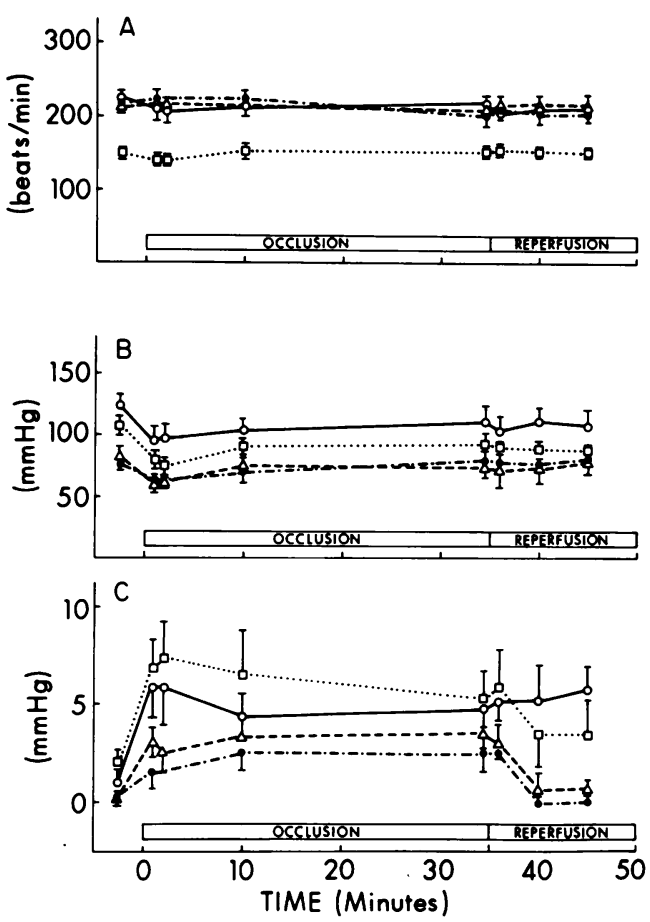

FIGURE 7 Alterations in heart rate (A), mean arterial pressure (B), and LVEDP (C) before occlusion and throughout coronary occlusion and subsequent reperfusion (horizontal bar). Values are mean \pm SEM for control animals $(O)$ and animals treated with either phentolamine $(\triangle)$, propranolol $(\square)$, or prazosin $(\bullet)$.

(Fig. 8). Stroke work was somewhat higher in control and propranolol-treated animals than those animals treated with the $\alpha$-receptor blocking agents. However, these differences in stroke work were not significant (Fig. 8). Thus, the marked antiarrhythmic effectiveness of $\alpha$-receptor blockade cannot be ascribed exclusively to reflex alterations in heart rate, or ventricular loading conditions. The modestly lower LVEDP in animals treated with $\alpha$-adrenergic blocking agents are more likely a consequence of the more regular rhythm than a cause of antiarrhythmic efficacy because reduction of LVEDP to $1 \pm 0.3 \mathrm{~mm} \mathrm{Hg}$ induced with nitroprusside did not stimulate the antiarrhythmic effect in control animals after coronary reperfusion $(291 \pm 18$ PVC, $n=2)$. Furthermore, maintenance of LVEDP at control levels after phentolamine $(8 \pm 1 \mathrm{~mm} \mathrm{Hg})$ by infusion of dextran $(n=2)$ did not diminish the antiarrhythmic effect during reperfusion (17 $\pm 3 \mathrm{PVC})$.

\section{DISCUSSION}

Reperfusion compared to sustained coronary occlusion induces malignant ventricular dysrhythmia, which appears to depend upon different, yet characteristic electrophysiological derangements (3). The dysrhythmia induced by coronary occlusion alone appears to be primarily a result of reentrant excitation secondary to 

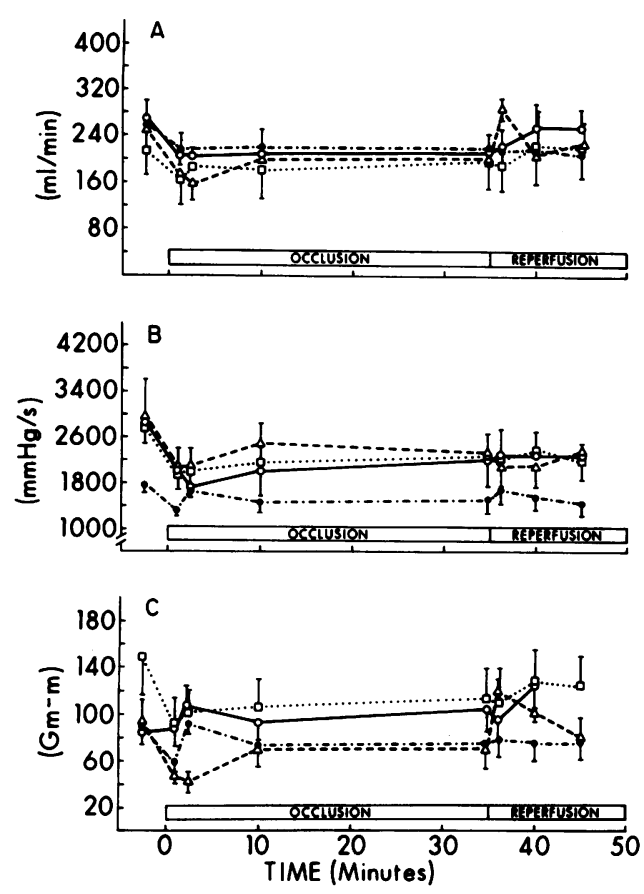

Figure 8 Alterations in cardiac output (A), peak dP/dt (B), and stroke work (C) before occlusion and throughout coronary occlusion and subsequent reperfusion (horizontal bar). Values are mean \pm SEM for control animals $(O)$ and animals treated with either phentolamine $(\triangle)$, propranolol $(\square)$, or prazosin (O).

increased conduction time throughout the ischemic region. It is accompanied by asynchronous depolarization, normal idioventricular rate, and shortening of the refractory period (3). On the other hand, dysrhythmia induced by reperfusion is dependent on different electrophysiological alterations because conduction time through myocardial regions is normal, and the rhythm is accompanied by synchronous depolarization and an elevated idioventricular rate (3). The present study was undertaken to evaluate contributions of $\alpha$ - and $\beta$-adrenergic neural influences on these two different types of dysrhythmia. The feline preparation was used in part because occlusion of the proximal LAD coronary artery results in reproducible ventricular dysrhythmia with a consistent time of onset, duration, frequency of ectopic activity, and mortality caused by $\operatorname{VF}(3,6)$. Furthermore, the feline preparation exhibits predictable and reproducible activation of both afferent and efferent autonomic nerves during myocardial ischemia. Reperfusion instituted 35 min after coronary occlusion elicits another type of ventricular dysrhythmia, which is characterized by a rapid onset time and brief duration (3). Although the antiarrhythmic effect of $\alpha$-blockade was most marked during early reperfusion, $\alpha$-adrenergic contributions to both types of dysrhythmia were evident because phentolamine and prazosin suppressed the number of PVC and prevented VF during occlusion and reperfusion. An alternative sympatholytic intervention, depletion of myocardial catecholamines by 6 hydroxydopamine pretreatment, elicited comparable protective effects even though $\beta$-adrenergic blockade was ineffective.

Left stellate nerve stimulation induced a marked increase in idioventricular rate dependent on $\beta$-adrenergic mechanisms before coronary occlusion but $\alpha$-adrenergic mechanisms after reperfusion judging from responses to propranolol and phentolamine. The apparent prominence of $\alpha$-adrenergic mechanisms during reperfusion may help to explain the ineffectiveness of $\beta$-receptor blockade in some cases on VF during myocardial ischemia $(4,6)$ compared to the marked effectiveness of myocardial catecholamine depletion (4, 5). After coronary occlusion, spontaneous increases in perfusion are prominent, mediated not only by collaterals but also possibly by diminished spasm of vessels within the ischemic bed itself. Under these conditions and particularly early after reperfusion the preponderance of $\alpha$-adrenergic mechanisms as contributors to electrophysiological alterations underlying malignant dysrhythmia may well militate against suppression by $\beta$-adrenergic blockade.

The increase in idioventricular rate (measured under conditions of vagal stimulation) induced by left stellate nerve stimulation during coronary occlusion alone was not altered by $\alpha$-receptor blockade but was abolished by $\beta$-receptor blockade (Figs. 4 and 5 ). Thus, the increased idioventricular rate did not appear to result from increased $\alpha$-adrenergic stimulation early during occlusion. On the other hand, $\alpha$-adrenergic blockade with either prazosin or phentolamine or depletion of myocardial catecholamines with 6-hydroxydopamine significantly attenuated both ventricular ectopic activity and VF induced by coronary occlusion (Table I). The differential effects of $\alpha$-blockade in the same setting on idioventricular rate as opposed to ventricular ectopic activity and VF may be a result of the nature of the stimulus. In the case of idioventricular rate, measurements during occlusion alone showed no increase. However, left stellate stimulation did augment the idioventricular rate in this setting. Because the augmentation was not blocked by phentolamine, its mechanism appeared to be neuronally induced $\beta$-stimulation. On the other hand, "acute" stellate ablation does not diminish ventricular ectopic activity or VF (5) induced by coronary occlusion. Furthermore, based on the data shown in Fig. 2 demonstrating no increase in idioventricular rate during coronary occlusion, it is likely that efferent left stellate nerve stimulation is not a consistent concomitant of ischemia in the preparation used in this study. Thus, these derangements cannot depend exclusively on neuronal stimulation. Because dysrhythmia induced by coronary occlusion alone is dependent 
on mechanisms that exacerbate reentrant excitation, including profound decreases in conduction velocity and alterations in regional refractory periods without increases in the idioventricular rate (3), presumably $\alpha$-adrenergic blockade during coronary occlusion favorably effects parameters other than idioventricular rate. Indeed methoxamine has been shown to induce conduction block in partially depolarized Purkinje fibers, a phenomenon partially reversed by phentolamine (23) and may thereby exacerbate dysrhythmia depending on reentrant excitation and may explain the profound antiarrhythmic influence of phentolamine during coronary occlusion in the present study. Catecholamines may of course, be released in myocardium because of ischemia per se (4), independent of local efferent neural activity. Their release may contribute to both the $\alpha$ - and $\beta$-adrenergic effects implicated in ventricular ectopic activity and VF early after coronary occlusion. The lack of an increase in idioventricular rate during coronary occlusion alone may also be caused in part by suppression of idioventricular rate by increased extracellular $\mathrm{K}^{+}(24)$, known to accumulate in the extracellular space early after coronary occlusion (25).

Because $\alpha$-adrenergic blocking agents such as phentolamine possess cholinergic blocking actions on isolated smooth muscle (26) inhibit the action of tyramine and potentiate the action of norepinephrine on isolated guinea pig atrium (27), and exhibit quinidine-like effects on canine Purkinje fibers at higher doses (28), experiments were performed to determine whether the protective electrophysiological influences observed in the present study were a result of specific $\alpha$-receptor blocking actions. Specificity of postsynaptic $\alpha$-adrenergic blockade is suggested by: ( $a$ ) The dramatic increase in idioventricular rate during coronary reperfusion induced by efferent left stellate nerve stimulation, a response obliterated by $\alpha$-adrenergic but not $\beta$-adrenergic blockade. This response could not have been caused by a local anesthetic action of phentolamine on nerve transmission because previous studies have demonstrated that in the cat, phentolamine at the same concentrations, does not alter sympathetic neural activity (13); (b) Induction of effects identical to those seen with phentolamine by prazosin, an $\alpha$-adrenergic blocker devoid of presynaptic blocking activity (29); (c) Increases in the idioventricular rate and dysrhythmia identical to those seen with stellate nerve stimulation induced by methoxamine during coronary reperfusion, responses not seen before coronary occlusion; and $(d)$ Effects identical to those seen with $\alpha$-receptor blockade but not $\beta$-receptor blockade induced by myocardial catecholamine depletion.

Alpha receptor blockade did not significantly alter regional coronary flow before, during, or after occlusion, suggesting that its protective influences were not caused by improved distribution of perfusion. Recently,
Powell and Fiegl (30) have demonstrated that under physiological conditions a decrease of carotid sinus pressure from 128 to $44 \mathrm{~mm} \mathrm{Hg}$ induces a $20 \%$ increase in coronary diastolic resistance mediated largely by $\alpha$-adrenergic mechanisms. However, it appears that under conditions of ischemia, $\alpha$-receptor mediated vasoconstriction is not prominent and that the hyperemia accompanying reperfusion is modulated primarily by factors independent of $\alpha$-adrenergic neural mechanisms.

Coronary occlusion in the dog induces a progressive increase in apparent $\beta$-adrenergic receptor number within $1 \mathrm{~h}$ after coronary occlusion (31). However, it has not been established whether or not these changes are associated with functional enhancement of $\beta$-receptor responsiveness. Receptor analyses in vitro after removal and incubation of ischemic tissue, may give results that are not necessarily analogous to those occurring in the intact ischemic heart because of the presence of potentially modulating metabolites in vivo. Furthermore, in a preliminary report (32) others have also demonstrated an increased role of $\alpha$-adrenoceptors in ischemic myocardium and both $\alpha$ - and $\beta$-adrenergic receptor stimulation appear to be important progenitors for the induction of VF in dogs treated with probucol (33).

The observed idioventricular rate during early reperfusion exceeded 180 beats $/ \mathrm{min}$, a rate far in excess of the peak rate of $70-80$ beats with maximal stellate ganglion stimulation under physiological conditions (34). Thus, abnormal impulse formation appeared to occur during early reperfusion. In dogs with complete atrioventricular block, intense stimulation of cardiac sympathetic nerves or splanchnic nerves to the adrenal medulla results occasionally in ventricular tachycardia with a rate in excess of 120 beats/min (35). This type of ventricular tachycardia is quite different from the physiological acceleration of idioventricular pacemakers induced by sympathetic nerve stimulation (35). Its rate is much more rapid and it begins and terminates abruptly usually outlasting the sympathetic stimulation (35). Analogous phenomena were seen during reperfusion in the present study. This type of abnormal ventricular activity may be a result of overdrive excitation, a phenomenon in which a driving stimulus in the presence of enhanced adrenergic activity leads to repetitive discharge (36). The higher idioventricular rate seen with reperfusion in the present study mediated via $\alpha$-adrenergic mechanisms rather than the physiological $\beta$-receptor activation influencing automaticity in normal Purkinje fibers and myocardium may be an example of the same type of abnormal automaticity. Previous studies in isolated Purkinje fibers have demonstrated an $\alpha$-adrenergic mediated decrease in spontaneous rate $(37,38)$, presumably because of inhibition of ${ }^{42} \mathrm{~K}$ uptake (38). However, the enhanced $\alpha$-adrenergic responsiveness in the present study results in an increase in 
idioventricular rate, suggesting that this inhibitory mechanism does not appear to be operative.

The rapid increase in idioventricular rate during coronary reperfusion may be related to delayed after depolarizations similar to those induced by digitalis (39) and secondary to a rapid increase in $\mathrm{Ca}^{++}$influx (36). Increased $\mathrm{Ca}^{++}$accumulation is seen in ischemic tissue that suddenly undergoes reperfusion (40), a phenomenon known to increase the magnitude of such depolarizations. Alpha-mediated adrenergic influences may magnify the inward calcium movement since in isolated depressed fibers the potentiation of slow response type depolarizations by catecholamines may be secondary to $\alpha$ rather than $\beta$-receptor stimulation (41).

Acute (as opposed to long-term) sympathetic denervation does not consistently decrease the incidence of VF induced by coronary occlusion (5) or by reperfusion. However, chronic cardiac sympathectomy and treatment with reserpine sufficient to deplete myocardial norepinephrine stores are both efficacious (5). These observations suggest that release of myocardial catecholamines independent of local efferent neural activity contributes to dysrhythmias during myocardial ischemia. Furthermore, others have reported failure of propranolol to decrease the incidence of VF during coronary reperfusion (42). The present results suggest that the deleterious electrophysiological alterations induced by ischemia and mediated by release of myocardial catecholamines are dependent to a considerable extent on $\alpha$-adrenergic mechanisms.

The dysrhythmias induced by coronary reperfusion appear to depend upon electrophysiological alterations different from those underlying dysrhythmias induced by coronary occlusion alone (3). Adrenergic mechanisms appear to contribute to both. The particular importance of $\alpha$-adrenergic influences under pathophysiological rather than physiological conditions may account for the promise of $\alpha$-adrenergic blockade in suppressing ventricular dysrhythmia accompanying ischemic heart disease in man (43).

\section{ACKNOWLEDGMENTS}

We appreciate the assistance of Ms. Linda Binns for preparation of the manuscript and the technical assistance of $\mathrm{Mr}$. Gerard Clarke and Ms. Diana Rankin. The authors also appreciate the statistical analyses performed by Dr. J. Philip Miller.

Research from the authors' laboratory was supported by National Institutes of Health grants HL 17646, Specialized Center of Research in Ischemic Heart disease, HL 21654, and Missouri Heart Association grant-in-aid.

\section{REFERENCES}

1. Bashe, W. J., N. Baba, M. D. Keller, J. C. Geer, and J. R. Anthony. 1975. Pathology of atherosclerotic heart disease in sudden death. II. The significance of myocardial infarction. Circulation. 52(Suppl. III): III-63-III-69.
2. Schaffer, W. A., and L. A. Cobb. 1975. Recurrent ventricular fibrillation and modes of death in survivors of out-of-hospital ventricular fibrillation. N. Engl. J. Med. 293: 259-262.

3. Penkoske, P. A., B. E. Sobel, and P. B. Corr. 1978. Disparate electrophysiological alterations accompanying dysrhythmia due to coronary occlusion and reperfusion in the cat. Circulation. 58: 1023-1035.

4. Corr, P. B., and R. A. Gillis. 1978. Autonomic neural influences on the dysrhythmias resulting from myocardial infarction. Circ. Res. 43: 1-9.

5. Ebert, P. A., R. B. Vanderbeek, R. J. Allgood, and D. C. Sabiston. 1970. Effect of chronic cardiac denervation on arrhythmias after coronary artery ligation. Cardiovasc. Res. 4: 141-147.

6. Corr, P. B., F. X. Witkowski, and B. E. Sobel. 1978 Mechanisms contributing to malignant dysrhythmias induced by ischemia in the cat. J. Clin. Invest. 61: 109119.

7. Williams, L. T., R. J. Lefkowitz, A. M. Watanabe, D. R. Hathaway, and H. R. Besch, Jr. 1977. Thyroid hormone regulation of $\beta$-adrenergic receptor number.J. Biol. Chem. 252: 2787-2789.

8. Sharma, V. K., and S. P. Banerjee. 1978. Alpha-adrenergic receptor in rat heart. Effects of thyroidectomy. J. Biol. Chem. 253: 5277-5279.

9. Limbird, L. E., and R. J. Lefkowitz. 1976. Adenylate cyclase-coupled beta adrenergic receptors: effect of membrane lipid-perturbing agents on receptor binding and enzyme stimulation by catecholamines. Mol. Pharmacol. 12: 559-567.

10. Hsueh, W., P. C. Isakson, and P. Needleman. 1977. Hormone selective lipase activation in the isolated rabbit heart. Prostaglandins. 13: 1073-1091.

11. Giotti, A., F. Ledda, and P. F. Mannaioni. 1973. Effects of noradrenaline and isoprenaline, in combination with $\alpha$ and $\beta$-receptor blocking substances on the action potential of cardiac Purkinje fibers. J. Physiol. 229: 99-113.

12. Pappano, A. J. 1971. Propranolol-insensitive effects of epinephrine on action potential repolarization in electrically driven atria of the guinea pig. J. Pharmacol. Exp. Ther. 117: 85-95.

13. DiMicco, J. A., T. Prestel, D. L. Pearle, and R. A. Gillis. 1977. Mechanism of cardiovascular changes produced in cats by activation of the central nervous system with picrotoxin. Circ. Res. 41: 446-451.

14. Dowdy, E. G., and K. Kaya. 1968. Studies of the mechanism of cariovascular responses to CI-581. Anesthesiology. 29: 931-943.

15. Fink, B. R., and A. Schoolman. 1963. Arterial blood acidbase balance in unrestrained walking cats. Proc. Soc. Exp. Biol. Med. 112: 328-330.

16. Corr, P. B., D. L. Pearle, J. R. Hinton, W. C. Roberts, and R. A. Gillis. 1976. Site of myocardial infarction. A determinant of the cardiovascular changes induced in the cat by coronary occlusion. Circ. Res. 39: 840-847.

17. Kostrzewa, R. M., and D. M. Jacobowitz. 1974. Pharmacological actions of 6-hydroxydopamine. Pharmacol. Rev. 26: $199-288$.

18. Passon, P. G., and J. D. Peuler. 1973. A simplified radiometric assay for plasma norepinephrine and epinephrine. Anal. Biochem. 51: 618-631.

19. Eliakim, M., S. Bellet, E. Tawil, and O. Muller. 1961. Effect of vagal stimulation and acetylcholine on the ventricle. Studies in dogs with complete atrioventricular block. Circ. Res. 9: 1372-1379.

20. Spear, J. F., and E. N. Moore. 1973. Influence of brief vagal and stellate nerve stimulation on pacemaker activity 
and conduction within the atrioventricular conduction system of the dog. Cir. Res. 32: 27-41.

21. Hering, H. E. 1924. Uber das angebliche vorkommen von acceleransfasen im halsvagus der saugetiere. Arch. Gesamte Physiol. Mens. Tiere (Pfluegers). 203: 100-109.

22. Barr, A. J., H. H. Goodnight, J. P. Sall, and J. P. Helwig. 1976. A User's Guide to SAS 76. Statistical Analysis System Inc., Raleigh, N. C.

23. Cranefield, P. F., B. F. Hoffman, and A. L. Wit. 1971. Block of conduction in partially depolarized cardiac Purkinje fibres induced by an $\alpha$-adrenergic agent. Nat. New Biol. 234: 159-160.

24. Vassalle, M., K. Greenspan, S. Jomain, and B. F. Hoffman. 1964. Effects of potassium on automaticity and conduction of canine hearts. Am. J. Physiol. 207: 334-340.

25. Harris, A. S. 1966. Potassium and experimental coronary occlusion. Am. Heart J. 71: 797-802.

26. Boyd, H., G. Burnstock, G. Campbell, A. Jowett, J. O’Shea, and M. Wood. 1963. The cholinergic blocking action of adrenergic blocking agents in the pharmacological analysis of autonomic innervation. Br. J. Pharmacol. 20: 418435.

27. Benfey, B. G., and K. Greeff. 1961. Interactions of sympathomimetic drugs and their antagonists on the isolated atrium. Br. J. Pharmacol. 17: 232-235.

28. Rosen, M. R., H. Gelband, and B. F. Hoffman. 1971. Effects of phentolamine on electrophysiologic properties of isolated canine Purkinje fibers. J. Pharmacol. Exp. Therap. 179: 586-593.

29. Cambridge, D., M. J. Davey, and R. Massingham. 1977. The pharmacology of antihypertensive drugs with special reference to vasodilators, alpha-adrenergic blocking agents and prazosin. Med.J. Aust. 2(Suppl): 2-6.

30. Powell, J. R., and E. O. Feigl. 1979. Carotid sinus reflex coronary vasoconstriction during controlled myocardial oxygen metabolism in the dog. Circ. Res. 44: 44-51.

31. Mukherjee, A., T. M. Wong, R. J. Lefkowitz, L. M. Buja, and J. T. Willerson. 1979. Influence of experimental canine myocardial ischemia on beta-adrenergic and muscarinic cholinergic receptors. Clin. Res. 27: 440A. (Abstr.)
32. Juhasz-Nagy, A., and D. M. Aviado. 1976. Increased role of alpha-adrenoceptors in ischemic myocardial zones. Physiologist. 19: 245. (Abstr.)

33. Elharrar, V., A. M. Watanabe, J. Molello, H. R. Besch, Jr., and D. P. Zipes. 1979. Adrenergically mediated ventricular fibrillation in probucol-treated dogs: roles of alpha and beta adrenergic receptors. PACE (Pacing Clin. Electrophysiol.). 2: 435-443.

34. Vassalle, M., J. H. Stuckey, and M. J. Levine. 1969. Sympathetic control of ventricular automaticity: role of the adrenal medulla. Am. J. Physiol. 217: 930-937.

35. Vassalle, M., R. E. Knob, M. Cummins, G. A. Lara, C. Castro, and J. H. Stuckey. 1977. An analysis of fast idioventricular rhythm in the dog. Circ. Res. 41: 218-226.

36. Vassalle, M., R. E. Knob, G. A. Lara, and J. H. Stuckev. 1976. The effect of adrenergic enhancement on overdrive excitation. J. Electrocardiol. (San Diego). 9: 335-343.

37. Rosen, M. R., A. J. Hordof, J. P. Ilvento, and P. Danilo, Jr. 1977. Effects of adrenergic amines on electrophysiological properties and automaticity of neonatal and adult canine Purkinje fibers. Circ. Res. 40: 390-400.

38. Posner, P., E. L. Farrar, and C. R. Lambert. 1976. Inhibitory effects of catecholamines in canine cardiac Purkinje fibers. Am. J. Physiol. 231: 1415-1420.

39. Vassalle, M., and E. Musso. 1976. On the mechanisms underlying digitalis toxicity in cardiac Purkinje fibers. Recent Adv. Stud. Card. Struct. Metab. 9: 355-376.

40. Walen, D. A., D. G. Hamilton, C. E. Ganote, and R. B. Jennings. 1974. Effect of a transient period of ischemia on myocardial cells. Am. J. Pathol. 74: 381-397.

41. Miura, Y., J. Inui, and H. Imamura. 1978. Alpha-adrenoceptor-mediated restoration of calcium-dependent potential in the partially depolarized rabbit papillary muscle. Naunyn-Schmiedebergs Arch. Pharmacol. 301: 201-205.

42. Sommers, H. M., and R. B. Jennings. 1972. Ventricular fibrillation and myocardial necrosis after transient ischemia. Arch. Intern. Med. 129: 780-789.

43. Gould, L., R. F. Gomprecht, and M. Zahir. 1971. Oral phentolamine for treatment of ventricular premature contractions. Br. Heart J. 33: 101-104. 